\title{
Structural insights into the mechanism defining substrate affinity in Arabidopsis thaliana dUTPase: the role of tryptophan 93 in ligand orientation
}

Noriko Inoguchi ${ }^{1}$, Kittichai Chaiseeda ${ }^{2}$, Mamoru Yamanishi ${ }^{2}$, Moon Ki Kim ${ }^{3}$, Yunho Jang ${ }^{4,6}$, Mamta Bajaj ${ }^{1}$, Catherine P. Chia ${ }^{1}$, Donald F. Becker ${ }^{5}$ and Hideaki Moriyama ${ }^{1 *}$

\begin{abstract}
Background: Deoxyuridine triphosphate nucleotidohydrolase (dUTPase) hydrolyzes dUTP to dUMP and pyrophosphate to maintain the cellular thymine-uracil ratio. dUTPase is also a target for cancer chemotherapy. However, the mechanism defining its substrate affinity remains unclear. Sequence comparisons of various dUTPases revealed that Arabidopsis thaliana dUTPase has a unique tryptophan at position 93, which potentially contributes to its degree of substrate affinity. To better understand the roles of tryptophan 93, A. thaliana dUTPase was studied.

Results: Enzyme assays showed that $A$. thaliana dUTPase belongs to a high-affinity group of isozymes, which also includes the enzymes from Escherichia coli and Mycobacterium tuberculosis. Enzymes from Homo sapiens and Saccharomyces cerevisiae are grouped as low-affinity dUTPases. The structure of the homo-trimeric A. thaliana dUTPase showed three active sites, each with a different set of ligand interactions between the amino acids and water molecules. On an a-helix, tryptophan 93 appears to keep serine 89 in place via a water molecule and to specifically direct the ligand. Upon being oriented in the active site, the C-terminal residues close the active site to promote the reaction.

Conclusions: In the high-affinity group, the prefixed direction of the serine residues was oriented by a positively charged residue located four amino acids away, while low-affinity enzymes possess small hydrophobic residues at the corresponding sites.
\end{abstract}

Keywords: Deoxyuridine triphosphate nucleotidohydrolase, Substrate affinity, Drug targets, Arabidopsis thaliana

\section{Background}

Deoxyuridine triphosphate nucleotidohydrolase (dUTPase; EC 3.6.1.23) is an important enzyme that prevents uracil misincorporation during de novo DNA synthesis [1]. It catalyzes the hydrolysis of dUTP to deoxyuridine monophosphate (dUMP) and inorganic pyrophosphate $[2,3]$, thereby maintaining an appropriate level of dUTP with respect to deoxythymidine triphosphate (dTTP)

\footnotetext{
*Correspondence: hmoriyama2@unl.edu

1 School of Biological Sciences, University of Nebraska-Lincoln, Lincoln, $N E$, USA

Full list of author information is available at the end of the article
}

levels [4]. Compromising dUTPase activity in fast-growing cells causes an imbalance in the dUTP-dTTP ratio that can cause uracil misincorporation into DNA [1]. Due to its role in fast-growth-specific cell death, dUTPase has been a target for cancer chemotherapy $[5,6]$.

Homo-trimeric dUTPases have three active sites, each of which consists of five conserved motifs [3] (Fig. 1a-c). An aspartate in motif 1 interacts with active site water molecules to stabilize the divalent cation cofactor $\mathrm{Mg}^{2+}$, which is important for enzymatic activity [7-9]. A serine in motif 2 interacts with the oxygen atom between $\alpha, \beta$-phosphate to induce a reaction-favorable orientation [10] (Ser 89 in Arabidopsis dUTPase). An aspartate in motif 3 activates 


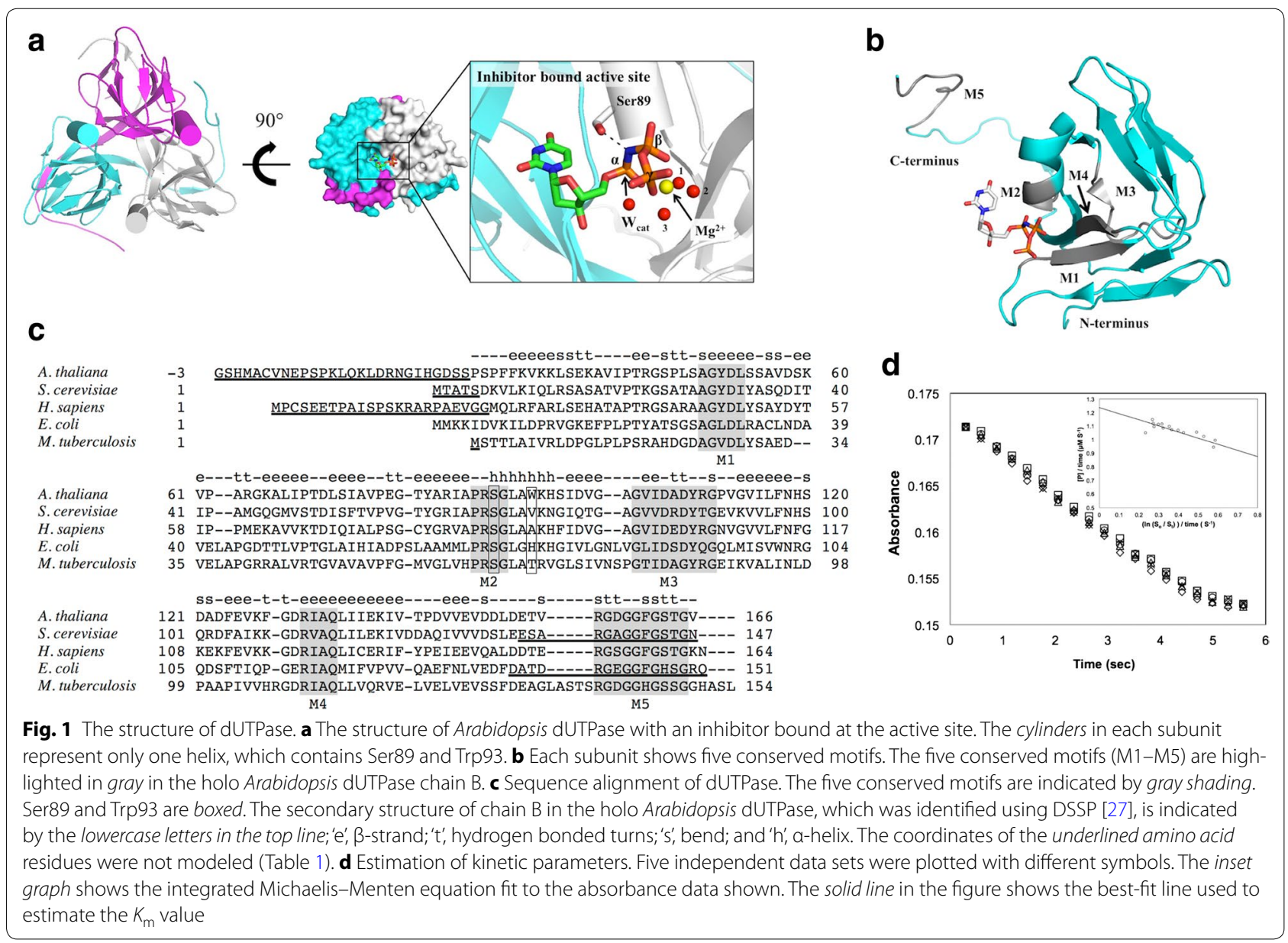

catalytic water [11]; a glutamine in motif 4 also interacts with the catalytic water (Wcat in Fig. 1a) and the ligand [9]. Interactions between ligands and residues in motif 5 help orient the ligand so that the $\alpha$ phosphate locates close to the catalytic water [12]. The homo-trimeric dUTPase kinetic mechanism has mainly been studied by multidimensional nuclear magnetic resonance (NMR) $[13,14]$, quench-flow experiments [15], and the mixed quantum mechanics/molecular mechanics $(\mathrm{QM} / \mathrm{MM})$ calculations [16]. These studies have revealed at least four distinct enzymatic steps including substrate binding, isomerization, hydrolysis, and release. However, the mechanism for defining the substrate affinity remains unclear.

To address the basis for substrate affinity differences among dUTPases, we chose those five dUTPases using two criteria, including (i) consistent measurement conditions of $\mathrm{K}_{\mathrm{m}}$ accompanied by (ii) reported X-ray structure of both apo and holo formats. We compared amino acid sequences as a function of substrate affinity, including in the analysis high-affinity isozymes (low $\mathrm{K}_{\mathrm{m}}$ ) from Escherichia coli [11], Mycobacterium tuberculosis [17], and Arabidopsis thaliana (thale cress; this study) [18] and low-affinity isozymes (high $\mathrm{K}_{\mathrm{m}}$ ) from Saccharomyces cerevisiae (yeast) [19] and Homo sapiens (human) [15] (Fig. 1c; Table 1).

We found that Arabidopsis dUTPase contains a unique tryptophan at the 93rd position, which is located between motifs 2 and 3 (Fig. 1c; Table 1). To identify the role of the 93rd tryptophan, we solved the structure of Arabidopsis dUTPase in its holo form. This homo-trimeric enzyme shows a unique set of interactions with ligands, amino acids, and water molecules at each active site. A comparison of the active sites reveals that tryptophan 93 seems to play a key role in guiding serine reorientation via a water molecule to orient the incoming ligand. In high-affinity dUTPases, the serine residue can be held in place in similar manner by a positively charged residue located four amino acids away. In contrast, low-affinity enzymes lack charges at the corresponding sites.

\section{Methods}

\section{dUTPase preparation}

Arabidopsis dUTPase was prepared as described previously [18, 20, 21]. Briefly, His-tagged Arabidopsis 
Table 1 Comparison of secondary structures and interactions at the ligand-binding site

\begin{tabular}{|c|c|c|c|c|c|}
\hline & A. thaliana & S. cerevisiae & H. sapiens & E. coli & M. tuberculosis \\
\hline PDB ID & $400 \mathrm{P}$ & $3 P 48$ & $2 \mathrm{HQU}$ & 1RN8 & $2 \mathrm{PY} 4$ \\
\hline Sequence similarity ${ }^{a}$ & Self & $56.2 \%$ & $63.9 \%$ & $35.5 \%$ & $39.2 \%$ \\
\hline$K_{\mathrm{m}}$ & $0.4 \pm 0.1 \mu \mathrm{M}$ & $13.2 \pm 0.6 \mu \mathrm{M}$ & $3.6 \pm 1.9 \mu \mathrm{M}$ & $\begin{array}{l}0.5 \mu \mathrm{M} \\
\text { (SE } 17 \%)\end{array}$ & $0.46 \pm 0.2 \mu \mathrm{M}$ \\
\hline a helix 1st res & S89 & S69 & $\mathrm{S} 86$ & $\mathrm{~S} 72$ & $\mathrm{~S} 65$ \\
\hline a helix 5th res & W93 & V73 & A90 & $\mathrm{H} 76$ & T69 \\
\hline a1st-WAT-a5th & Present & Absent & Absent & Absent & Absent \\
\hline References & This study & Tchigvintsev et al. [19] & Toth et al. [15] & Barabas et al. [11] & Pecsi et al. [17] \\
\hline
\end{tabular}

a1st-WAT-a5th refers to the presence of an interaction among the first residue of the a helix, a water molecule, and the fifth residue of the a helix

a Sequence similarities between Arabidopsis and each species were calculated by using SIM [28] on the ExPASy server (http://www.expasy.org)

dUTPase was purified via Ni-NTA chromatography from the cleared lysate of E. coli JM103 (DE3) cells. The tag was removed by thrombin cleavage. The resulting dUTPase includes three extra amino acids, Gly-Ser-His, at the amino-terminus (Fig. 1c).

\section{dUTPase activity assay}

The enzymatic activity assay was performed using cresol red, and the $K_{m}$ values were calculated using the integrated Michaelis-Menten method [22, 23] with a stopped-flow instrument (Hi-Tech SF-61DX2, TgK Scientific, Bradford-on-Avon, UK) equipped with a photodiode array detector. The assay solution contained $100 \mathrm{mM}$ $\mathrm{KCl}, 5 \mathrm{mM} \mathrm{MgCl}_{2}$, and $0.25 \mathrm{mM}$ bicine at $\mathrm{pH}$ 7.6. dUTPase, to a final concentration of $50 \mathrm{nM}$, was rapidly mixed with 1-5 $\mu \mathrm{M}$ dUTP solutions in the stopped-flow system, and absorbance was monitored at $573 \mathrm{~nm}$ (Fig. 1d).

\section{Crystallization}

Crystals of holo Arabidopsis dUTPase were grown by vapor diffusion with the hanging drop method using $2 \mathrm{M}$ ammonium sulfate as a precipitant in $50 \mathrm{mM}$ Tris$\mathrm{HCl}$ at $\mathrm{pH} 7.4$ and $5 \mathrm{mM}$ of the non-hydrolyzable ligand analog $2^{\prime}$-deoxyuridine $\quad 5^{\prime}$ - $[(\alpha, \beta)$-imido $]$-triphosphate (dUpNHpp; Jena Bioscience, Jena, Germany) along with $5 \mathrm{mM} \mathrm{MgSO}_{4}$ (Table 2) [18, 20, 21].

\section{X-ray diffraction data collection, structural analysis, and structural mining}

Diffraction data were collected from a single holo dUTPase crystal at the Advanced Photon Source (Argonne, IL, USA) sector 14-BM-C. The data collection and refinement statistics are shown in Table 2. While the holo crystal diffracted beyond $1.2 \AA$ resolution, we only used reflections up to $1.5 \AA$ resolution to keep the redundancy more than five and the linear $\mathrm{R}$ factor less than 0.6. To increase the resolution of the structure in apo formats, we further refined the apo structure (PDB ID, 2P9O) with previously collected data by PHENIX [24]; the updated apo structure was used as the starting model for the structure in the holo format. The structure of the holo enzyme was also refined by PHENIX using the same Rfree flag assignment used in apo structure refinement. We deposited the apo and holo structures in PDB with the IDs 4OOQ and 4OOP, respectively. The COOT [25], PISA [26], and PyMOL (Schrödinger, San Diego, CA) software packages were used for structural mining and graphical presentation.

\section{Results and discussion}

\section{Enzymatic activity and crystallization}

The Arabidopsis dUTPase showed enzymatic activity, with estimated $\mathrm{K}_{\mathrm{m}}$ and $\mathrm{V}_{\max }$ values of $0.4 \pm 0.1 \mu \mathrm{M}$ and $1.2 \pm 0.05 \mu \mathrm{M} \mathrm{s}^{-1}$, respectively, at $\mathrm{pH} 7.6$ and $25{ }^{\circ} \mathrm{C}$ (Fig. 1d). Therefore, the enzyme belongs to the high-substrate-affinity group, which also includes the E. coli and M. tuberculosis proteins (Table 1).

Crystallization of the holo Arabidopsis dUTPase was performed using the non-hydrolysable dUTP analog dUpNHpp and ammonium sulfate as the precipitant. In the apo Arabidopsis dUTPase, taurine was an indispensable additive for the growth of single crystals [18]. In the holo format, the growth of single crystals was not dependent on the presence of taurine. The protein formed needle clusters in the absence of dUpNHpp and taurine.

\section{Arabidopsis dUTPase structure}

The refined Arabidopsis dUTPase structure showed a trimeric structure (Figs. 1a, 2a; Table 2). The first 24 $\mathrm{N}$-terminal residues of all of the subunits were equally disordered. However, there were notable differences in the interpretable C-terminal domains when all of the active sites had a bound ligand (Fig. 2b). Next, we used PISA to assess the effects of crystal packing on the different C-terminal lengths [26]. Chains A and C interacted 
Table 2 Data collection and structural refinements of Arabidopsis dUTPase

\begin{tabular}{|c|c|c|}
\hline Parameters & Apo dUTPase & Holo dUTPase \\
\hline PDB ID & $400 Q$ & $400 P$ \\
\hline System & Orthorhombic & Orthorhombic \\
\hline Space group & P212121 & P212121 \\
\hline \multicolumn{3}{|l|}{ Unit cell dimensions $(\AA)$} \\
\hline a & 69.9 & 70.1 \\
\hline$b$ & 70.6 & 70.7 \\
\hline c & 75.0 & 75.1 \\
\hline \multicolumn{3}{|l|}{ Data collection } \\
\hline Wavelength $(\AA)$ & 1.542 & 0.978 \\
\hline Resolution (scaling) range $(\AA)$ & $20.34-2.00(2.1-2.0)$ & $31.97-1.5(1.55-1.5)$ \\
\hline No. of observed reflections & 209,897 & 360,703 \\
\hline \multicolumn{2}{|c|}{ No. of unique reflections used 25,874} & $59,499(5804)$ \\
\hline $1 / s()$ & $41.6(18.5)$ & $46.4(8.6)$ \\
\hline Completeness (\%) & $99.2(98.5)$ & $95.0(90.0)$ \\
\hline$R_{\text {merge }}(\%)$ & $7.6(17.0)$ & $7.0(32.0)$ \\
\hline \multicolumn{3}{|l|}{ Refinement } \\
\hline Resolution range $(\AA)$ & $20.34-2.00(2.1-2.0)$ & $31.97-1.5(1.55-1.5)$ \\
\hline $\mathrm{R}_{\text {work }}(\%)$ & 14.8 & 17.3 \\
\hline $\mathrm{R}_{\text {free }}(\%)$ & 19.6 & 20.8 \\
\hline No. of non-hydrogen atoms & 3287 & 3658 \\
\hline No. of water molecules & 345 & 552 \\
\hline \multicolumn{3}{|l|}{ RMS deviations from ideal values } \\
\hline Bond lengths $(\AA)$ & 0.007 & 0.007 \\
\hline Bond angles $\left(^{\circ}\right)$ & 1.08 & 1.16 \\
\hline Mean $B$ value $\left(\AA^{2}\right)$ & 22.0 & 20.0 \\
\hline \multicolumn{3}{|l|}{ Interpretable residues (out of 166) } \\
\hline Chain A & $26-156$ & $25-157$ \\
\hline Chain B & $25-154$ & $25-166$ \\
\hline Chain C & $26-152$ & $26-152$ \\
\hline
\end{tabular}

Values in parentheses correspond to the highest-resolution shell

$R_{\text {merge }}=\Sigma\left|I_{\text {obs }}-\langle I\rangle\right| \Sigma I_{\text {obs }}$, where $I_{\text {obs }}$ and $\langle I\rangle$ are the observed intensity and the mean intensity of the reflection, respectively

$R_{\text {work }}=\Sigma|| F_{\text {obs }}|-| F_{\text {calc }}|| / \Sigma\left|F_{\text {obs }}\right|$

$R_{\text {free }}$ values are collected for a randomly selected $5 \%$ of the data that was excluded from the refinement

with neighboring subunits (Additional file 1: Figure S1), while chain B did not. Since $\alpha$ carbon positions between chain $\mathrm{B}$ and $\mathrm{C}$ are identical, crystal packing apparently contributes to the variety in the chain A C-terminal length.

\section{Three apo-holo common waters accommodate the substrate}

Because the C-terminal residues are involved in ligand interactions, the environment of the ligand-binding site was analyzed (Additional file 2: Table S1 and Additional file 3: Figure S2). Aside from the three ordered water molecules that interact with the magnesium ion, there were three additional ordered water molecules (Fig. 2c) that interact with the ligand at the nitrogenous base oxygen atom $\mathrm{O}_{4}$, the $\alpha$-phosphate oxygen atom, and the $\beta$-phosphate oxygen atom. When the active sites of both the apo and holo forms are superimposed, all of the apo active sites have three water molecules at similar locations as those commonly found in the holo form. These data suggest that the coordination of these water molecules is necessary for initial ligand binding at the active site.

\section{Replacement of ligand-associated water with C-terminal residues reorients the ligand}

Ligand 1 bound to active site 1 involves the shortest interpretable C-terminus (Fig. 2d, active site 1 ) and has the most interactions with water molecules. Ligand 2 in active site 2 involves a medium-length interpretable $\mathrm{C}$-terminus, and has the fewest interactions with water molecules and amino acid residues. Ligand 2 has the highest average $\mathrm{B}$-factor among the 3 ligands, namely $42.8 \AA^{2}$ as calculated by COOT. A likely explanation for this result is that the $\mathrm{C}$-terminal residues involved in this active site have the most interactions with neighboring subunits due to crystal packing, as discussed above (Additional file 1: Figure S1). Superimposition of the ligand 1 and 2 binding sites (Fig. 2d, active sites 1 and 2) shows that the ligand 1-interacting water molecule (indicated by a green arrow in Fig. 2d) is located at a coordinate similar to that of the Arg156 amino group in the ligand 2 binding site. The electron density map does not support the presence of the corresponding Arg156 coordinate in the ligand 1 binding site. We interpret these data as ligand 1 being in a pre-ordered state, such that the bound ligand is reoriented by the replacement of the ligand-water interaction with the ligand-Arg156 interaction.

This "interaction-replacement" phenomenon is also observed in the ligand 3 binding site (red and black arrows in Fig. 2d, active sites 2 and 3). This binding site involves the completed $\mathrm{C}$-terminus and has the largest number of ligand-amino acid interactions. A superimposition of all of the ligand-binding sites shows that Arg156 undergoes a structural change to interact with the water molecule ordered by the magnesium ion in the holo form. Some ligand-water molecule interactions found in both the ligand 1 and 2 binding sites are replaced by ligandSer163 or ligand-Thr164 interactions in the ligand 3 binding site.

We compared ligand coordinates focusing between ligand 1 and 3 binding sites. It was because the C-terminal residues involved in the ligand 2 binding site (chain A residues from 145 to 152) had interactions with four neighboring residues, and resulted the structural 


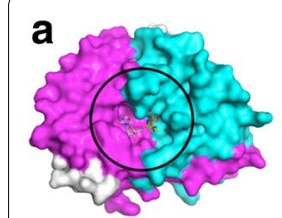

Active site 1

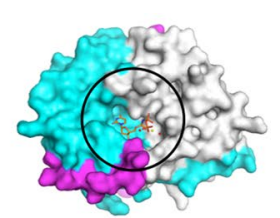

Active site 2

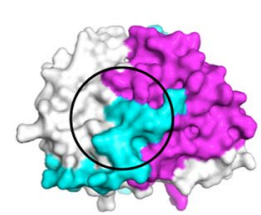

Active site 3

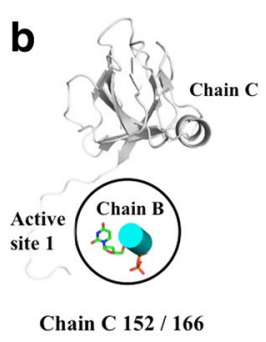

d

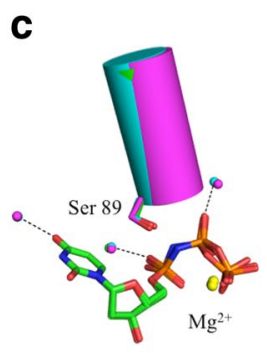

Holo active sites
8

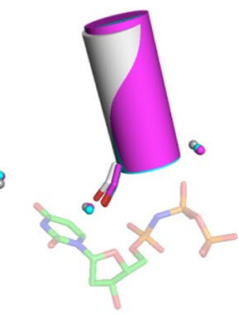

Apo active sites

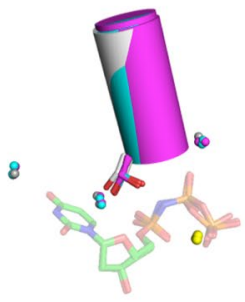

Holo and Apo

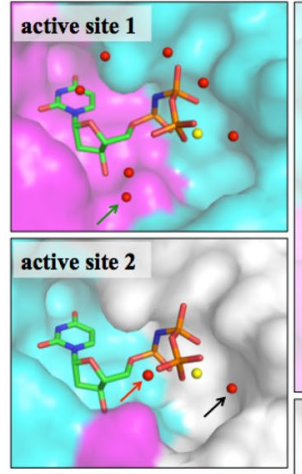

active site 3

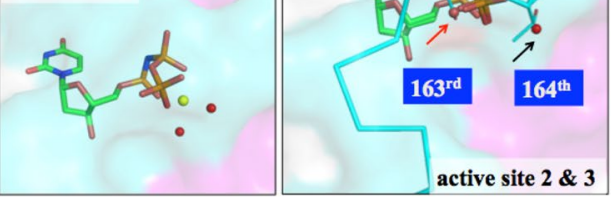

Fig. 2 The structures of the apo and holo forms of Arabidopsis dUTPase. a Surface presentation of the dUTPase trimer. Circles indicate the active sites. Chains A, B, and C are colored in magenta, cyan, and gray, respectively. $\mathbf{b}$ The structure of each active site is composed of three subunits. Each diagram contains a ligand, an a-helix carrying Ser89 and Trp93, and a subunit that provides the C-terminal residues to the active site in a cartoon model. The lengths of the $\mathrm{C}$-termini of the subunits are different. $\mathbf{c}$ Apo and holo common water molecules at the active site. Stick models represent the ligand and the ligand-interacting serine residue (S89). $\mathbf{d}$ Ligand interaction-replacement by water molecules and amino acids. The water molecules indicated by the arrow are initially associated with the ligand and later potentially replaced with C-terminal amino acids

difference in the $\mathrm{C}$-termini residues comparing from other subunits, chain B and C (Additional file 1: Figure $\mathrm{S} 1$ ). It appears that the ligand coordinate in the ligand 3 binding site appears to be engaged in the more stable orientation in terms of increased ligand-amino acid interactions. Additionally, the ligand coordinate is in the most favorable position for nucleophilic attack. The $\gamma$-phosphate group of ligand 3 occupies a position that is different from those of the other two ligands. A comparison of the ligand 3 and 1 coordinates relative to the catalytic water shows that the ligand $3 \alpha$-phosphate is closer to the catalytic water by approximately $0.3 \AA$ ( $W_{\text {cat }}$ in Fig. 1a, inhibitor-bound active site).

\section{Roles of Trp93 in Arabidopsis dUTPase ligand binding}

Serine 89 of motif 2 in Arabidopsis dUTPase is an important residue for maintaining a reaction-favorable ligand orientation at the active site (Fig. 2c). It undergoes a conformational change between the apo and holo forms to interact with the oxygen atom between $\alpha, \beta$-phosphate [10]. This serine side chain flipping is observed in the obtained Arabidopsis dUTPase structure (Figs. 2c, 3a). This rearrangement is commonly observed in all active sites of the compared dUTPases except for one of the active sites in yeast dUTPase.

Tryptophan 93 of Arabidopsis dUTPase may play an important role in orienting serine 89 in the holo form (Fig. 2c). In the apo form, all of the tryptophan 93 sidechain coordinates were oriented upwards; thus, all of the tryptophan $93 \mathrm{~N}^{\varepsilon 1}$ atoms were located away from the serine $89 \mathrm{O}^{\gamma}$. These are likely due to crystal-packing induced hydrophobic interactions with proline 46 in neighboring subunits. In contrast, the tryptophan 93 side chain in the holo-form chain B had different coordinates compared with those in other two subunits. It is located such that the tryptophan $93 \mathrm{~N}^{\varepsilon 1}$ was closer to the serine $89 \mathrm{O}^{\gamma}$. Although tryptophan 93 in the holo form may make the same hydrophobic interactions with proline 46 


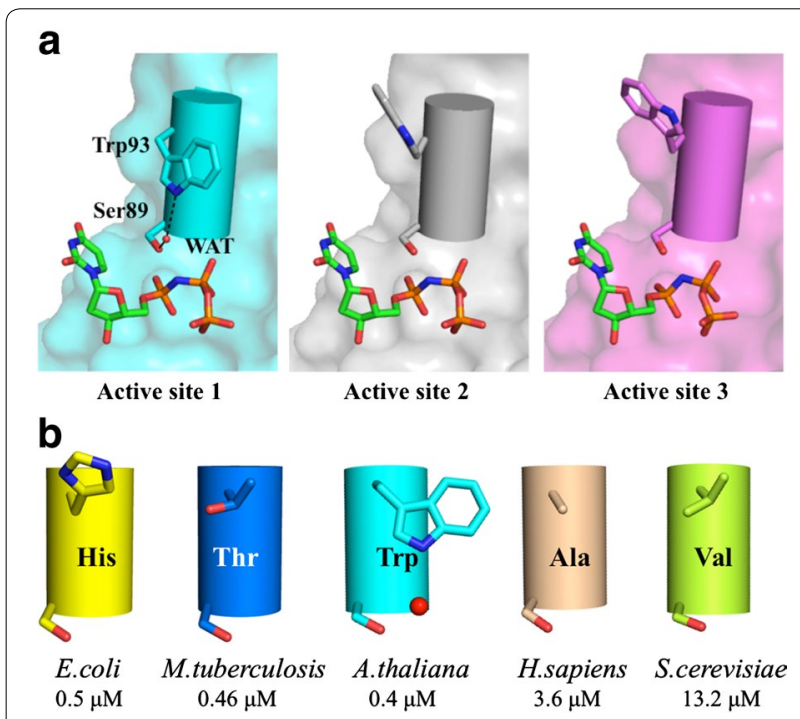

Fig. 3 Interactions between serine 89 and tryptophan 93 in Arabidopsis dUTPase. a Location of the Trp93 side chain in each subunit at different stages of substrate orientation. b Comparison of amino acids in other dUTPases that correspond to Ser 89 and Trp 93 in Arabidopsis dUTPase

in neighboring subunit, this particular orientation was likely due to the presence of a nearby water molecule, which bridges its interaction with serine 89 . This serine 89-water-tryptophan 93 interaction was only found in chain $\mathrm{B}$. This is part of the ligand 1 binding site, which involves the shortest interpretable C-terminus.

Together with the finding that ligand 1 is likely in a pre-ordered state for the reaction, we assume that tryptophan 93 acts as a key residue for initial ligand orientation at the active site by promoting serine 89 side-chain coordinate changes by interacting with the ordered water molecule.

Molecular mechanism for the differences in ligand affinity Arabidopsis dUTPase belongs to the high-affinity group, along with the E. coli and Mycobacterium dUTPases (Table 1; Fig. 2c). Tryptophan 93 of Arabidopsis dUTPase holds serine 89 in place via a water molecule and forms a favorable conformation for substrate binding. It appears that the high-affinity dUTPases from species such as $E$. coli and Mycobacterium have charged or polar aminoacid substitutions corresponding to tryptophan 93 in Arabidopsis dUTPase. In contrast, the low-affinity dUTPases from humans and yeast have non-polar amino-acid substitutions. Additionally, chain $\mathrm{C}$ of the yeast dUTPase has the motif 2 serine residue whose side-chain oxygen atom is located away from the nitrogen atom between $\alpha, \beta$-phosphate, and yeast dUTPase has the highest $K_{m}$ value among the five dUTPases compared in this study.
These data suggest that the amino-acid substitution affects the hydration state at the active site and may influence ligand-binding affinity.

\section{Conclusions}

The structure of Arabidopsis dUTPase has been analyzed. Interestingly, this homotrimeric enzyme shows varying binding site environments with respect to their types of ligand interactions. Additionally, the tryptophan 93 substitution seems to use ordered water molecules to aid in coordinating Ser89 for initial ligand binding.

\section{Additional files}

Additional file 1: Figure S1. Effect of crystal packing on C-terminal residue coordinates.

Additional file 2: Table S1. List of common ligand interactions.

Additional file 3: Figure S2. Common ligand interactions at the active site.

\section{Authors' contributions}

$\mathrm{NI}$ and MB carried out the protein crystallography. $\mathrm{NI}, \mathrm{KC}$, and MY carried out the kinetic analysis. NI and YJ carried out the molecular dynamics and structural mining. HM, DB, CPC, MKK conceived the study, designed the experiments, analyzed the data and wrote the manuscript. All authors read and approved the final manuscript.

\section{Author details}

${ }^{1}$ School of Biological Sciences, University of Nebraska-Lincoln, Lincoln, NE, USA. ${ }^{2}$ Department of Chemistry, University of Nebraska-Lincoln, Lincoln, NE, USA. ${ }^{3}$ School of Mechanical Engineering, Sungkyunkwan University, 300 Cheoncheon, Suwon, South Korea. ${ }^{4}$ Department of Mechanical and Industrial Engineering, University of Massachusetts, Amherst, MA, USA. ${ }^{5}$ Department of Biochemistry, University of Nebraska-Lincoln, Lincoln, NE, USA. ${ }^{6}$ Present Address: Virology, Surveillance and Diagnostic Branch, Influenza Division, Centers for Disease Control and Prevention, Atlanta, GA, USA.

\section{Acknowledgements}

We thank the Arabidopsis Biological Resource Center at the Ohio State University for providing us with the dUTPase clone. We also thank Dr. Javier Seravalli in the Dept. of Biochemistry at UNL for his contributions to the stopped-flow activity assays. This research used resources from the Advanced Photon Source, a US Department of Energy (DOE) Office of Science User Facility operated for the DOE Office of Science by Argonne National Laboratory under Contract No. DE-AC02-06CH11357.

\section{Competing interests}

The authors declare that they have no competing interests.

Received: 2 September 2015 Accepted: 26 November 2015

Published online: 15 December 2015

\section{References}

1. Hogrefe HH, Hansen CJ, Scott BR, Nielson KB. Archaeal dUTPase enhances PCR amplifications with archaeal DNA polymerases by preventing dUTP incorporation. Proc Natl Acad Sci USA. 2002;99(2):596-601. doi:10.1073/ pnas.012372799.

2. Mol CD, Harris JM, Mclntosh EM, Tainer JA. Human dUTP pyrophosphatase: uracil recognition by a beta hairpin and active sites formed by three separate subunits. Structure. 1996;4(9):1077-92. 
3. Vertessy BG, Toth J. Keeping uracil out of DNA: physiological role, structure and catalytic mechanism of dUTPases. Acc Chem Res. 2009;42(1):97106. doi:10.1021/ar800114w.

4. Greenberg GR, Somerville RL. Deoxyuridylate kinase activity and deoxyuridinetriphosphatase in Escherichia coli. Proc Natl Acad Sci USA. 1962;48:247-57.

5. Ladner RD. The role of dUTPase and uracil-DNA repair in cancer chemotherapy. Curr Protein Pept Sci. 2001;2(4):361-70.

6. Hassan M, Watari H, AbuAlmaaty A, Ohba Y, Sakuragi N. Apoptosis and molecular targeting therapy in cancer. Biomed Res Int. 2014;2014:150845. doi:10.1155/2014/150845.

7. Oliveros M, Garcia-Escudero R, Alejo A, Vinuela E, Salas ML, Salas J. African swine fever virus dUTPase is a highly specific enzyme required for efficient replication in swine macrophages. J Virol. 1999;73(11):8934-43.

8. Zhang Y, Moriyama H, Homma K, Van Etten JL. Chlorella virus-encoded deoxyuridine triphosphatases exhibit different temperature optima. J Virol. 2005;79(15):9945-53.

9. Takacs E, Nagy G, Leveles I, Harmat V, Lopata A, Toth J, et al. Direct contacts between conserved motifs of different subunits provide major contribution to active site organization in human and mycobacterial dUTPases. FEBS Lett. 2010;584(14):3047-54. doi:10.1016/j.febslet.2010.05.018.

10. Palmen LG, Becker K, Bulow L, Kvassman JO. A double role for a strictly conserved serine: further insights into the dUTPase catalytic mechanism. Biochemistry. 2008:47(30):7863-74. doi:10.1021/bi800325j.

11. Barabas O, Pongracz V, Kovari J, Wilmanns M, Vertessy BG. Structural insights into the catalytic mechanism of phosphate ester hydrolysis by dUTPase. J Biol Chem. 2004;279(41):42907-15. doi:10.1074/jbc. M406135200.

12. Varga B, Barabas O, Kovari J, Toth J, Hunyadi-Gulyas E, Klement E, et al. Active site closure facilitates juxtaposition of reactant atoms for initiation of catalysis by human dUTPase. FEBS Lett. 2007:581(24):4783-8. doi:10.1016/j.febslet.2007.09.005.

13. Dubrovay Z, Gaspari Z, Hunyadi-Gulyas E, Medzihradszky KF, Perczel A Vertessy BG. Multidimensional NMR identifies the conformational shift essential for catalytic competence in the 60-kDa Drosophila melanogaster dUTPase trimer. J Biol Chem. 2004;279(17):17945-50. doi:10.1074/jbc. M313644200.

14. Barabas O, Nemeth V, Bodor A, Perczel A, Rosta E, Kele Z, et al. Catalytic mechanism of alpha-phosphate attack in dUTPase is revealed by X-ray crystallographic snapshots of distinct intermediates, 31P-NMR spectroscopy and reaction path modelling. Nucleic Acids Res. 2013;41(22):1054255. doi:10.1093/nar/gkt756.

15. Toth J, Varga B, Kovacs M, Malnasi-Csizmadia A, Vertessy BG. Kinetic mechanism of human dUTPase, an essential nucleotide pyrophosphatase enzyme. J Biol Chem. 2007;282(46):33572-82. doi:10.1074/jbc. M706230200.
16. Lopata A, Jambrina PG, Sharma PK, Brooks BR, Toth J, Vertessy BG, Rosta E. Mutations decouple proton transfer from phosphate cleavage in the dutpase catalytic reaction. ACS Catal. 2015;5:3225-37.

17. Pecsi I, Leveles I, Harmat V, Vertessy BG, Toth J. Aromatic stacking between nucleobase and enzyme promotes phosphate ester hydrolysis in dUTPase. Nucleic Acids Res. 2010;38(20):7179-86. doi:10.1093/nar/gkq584

18. Bajaj M, Moriyama H. Purification, crystallization and preliminary crystallographic analysis of deoxyuridine triphosphate nucleotidohydrolase from Arabidopsis thaliana. Acta Crystallogr, Sect F: Struct Biol Cryst Commun. 2007;63(Pt 5):409-11. doi:10.1107/S1744309107016004

19. Tchigvintsev A, Singer AU, Flick R, Petit P, Brown G, Evdokimova E, et al. Structure and activity of the Saccharomyces cerevisiae dUTP pyrophosphatase DUT1, an essential housekeeping enzyme. Biochem J. 2011;437(2):243-53. doi:10.1042/BJ20110304.

20. Homma K, Moriyama H. Crystallization and crystal-packing studies of Chlorella virus deoxyuridine triphosphatase. Acta Crystallogr, Sect F: Struct Biol Cryst Commun. 2009;65(Pt 10):1030-4. doi:10.1107/ S1744309109034459.

21. Badalucco L, Poudel I, Yamanishi M, Natarajan C, Moriyama H. Crystallization of Chlorella deoxyuridine triphosphatase. Acta Crystallogr Sect F Struct Biol Cryst Commun. 2011;67(Pt 12):1599-602. doi:10.1107/ S1744309111038097.

22. Larsson G, Nyman PO, Kvassman JO. Kinetic characterization of dUTPase from Escherichia coli. J Biol Chem. 1996:271(39):24010-6.

23. Vertessy BG. Flexible glycine rich motif of Escherichia coli deoxyuridine triphosphate nucleotidohydrolase is important for functional but not for structural integrity of the enzyme. Proteins. 1997;28(4):568-79.

24. Adams PD, Afonine PV, Bunkoczi G, Chen VB, Davis IW, Echols N, et al. PHENIX: a comprehensive Python-based system for macromolecular structure solution. Acta Crystallogr Sect D Biol Crystallogr. 2010;66(Pt 2):213-21. doi:10.1107/S0907444909052925.

25. Emsley P, Cowtan K. Coot: model-building tools for molecular graphics. Acta Crystallogr Sect D Biol Crystallogr. 2004;60(Pt 12 Pt 1):2126-32. doi:10.1107/S0907444904019158.

26. Krissinel $E_{1}$ Henrick K. Inference of macromolecular assemblies from crystalline state. J Mol Biol. 2007;372(3):774-97. doi:10.1016/j.jmb.2007.05.022.

27. Kabsch W, Sander C. Dictionary of protein secondary structure: pattern recognition of hydrogen-bonded and geometrical features. Biopolymers. 1983;22(12):2577-637. doi:10.1002/bip.360221211.

28. Huang X, Miller W. A time-efficient, linear-space local similarity algorithm. Advances in Applied Mathematics. 1991;12:337-357. doi:10.1016/0196-8858(91)90017-D.

\section{Submit your next manuscript to BioMed Central and we will help you at every step:}

- We accept pre-submission inquiries

- Our selector tool helps you to find the most relevant journal

- We provide round the clock customer support

- Convenient online submission

- Thorough peer review

- Inclusion in PubMed and all major indexing services

- Maximum visibility for your research

Submit your manuscript at www.biomedcentral.com/submit
O Biomed Central 